\title{
PRIORIDADES COMPETITIVAS Y ÁREAS DE DECISIÓN ESTRATÉGICA EN LA MANUFACTURA. UN ESTUDIO EMPÍRICO EN EL SECTOR DE ALIMENTOS*
}

\author{
Laura E. Castaño** \\ Jorge A. Vivares"*: \\ William Sarache ${ }^{* * * * * *}$
}

* doi: 10.11144/Javeriana.cao30-55.pcade. Este artículo presenta los hallazgos de un estudio empírico realizado en 49 empresas de alimentos del Departamento de Caldas, Colombia, financiado por la Universidad Nacional de Colombia. El artículo se recibió el 14/12/2016 y se aprobó el 25/11/2017. Sugerencia de citación: Castaño, L. E., Vivares, J. A. y Sarache, W. (2017). Prioridades competitivas y áreas de decisión estratégica en la manufactura. Un estudio empírico en el sector de alimentos. Cuadernos de Administración, 30(55), 67-96. http:// dx.doi.org/10.11144/Javeriana.cao30-55.pcade.

** Magister en Ingeniería Industrial de la Universidad Nacional de Colombia, Manizales, Colombia, 2017. Administradora de Empresas de la Universidad Nacional de Colombia, Manizales, Colombia, 2014.

Correo electrónico: laecastanogo@unal.edu.co

*** Doctor en Ingeniería de la Universidad Nacional de Colombia, Manizales, Colombia, 2018. Magister en Ingeniería Industrial de la Universidad Nacional de Colombia, Manizales, Colombia, 2013.

Correo electrónico: javivaresv@unal.edu.co

**** Doctor en Ciencias Técnicas de la Universidad Central de las Villas, Santa Clara, Cuba, 2003. Profesor Titular en el Departamento de Ingeniería Industrial en la Universidad Nacional de Colombia, Manizales, Colombia.

Correo electrónico:wasarachec@unal.edu.co 


\section{Prioridades competitivas y áreas de decisión estratégica en la manufactura. Un estudio empírico en el sector de alimentos}

\section{RESUMEN}

El objetivo de este artículo es contrastar algunas hipótesis relacionadas con las prioridades competitivas (PC) y las áreas de decisión estratégica $(A D E)$ en estrategia de manufactura (EM). Se identificó un conjunto ampliado de PC respecto a los estudios previos y se aporta evidencia empírica en el sector de alimentos. Para tal fin se realizó un estudio en 49 empresas en el Departamento de Caldas, Colombia. En las PC se identificó su importancia relativa y el enfoque de gestión dominante (cono de arena o trade-off). En cuanto a las ADE se evaluó la relación de su grado de desarrollo con el desempeño en las PC. Los resultados corroboran diversas posturas teóricas y demuestran que no existe un único camino para abordar la estrategia de manufactura.

Palabras clave: Estrategia de manufactura, estrategia de operaciones, prioridades competitivas, áreas de decisión estratégica, modelos cono de arena y trade-off. Clasificación JEL: D2, M11, L1

Competitive priorities and strategic decision areas in manufacturing. An empirical study in the food sector

\section{ABSTRACT}

The objective of this article is to test some theoretical hypothesis related to the competitive priorities (CP) and strategic decision areas (SDA) in manufacturing strategy (MS). As a main contribution it was identified a complete set of PC respect to the previous studies; also, empirical evidence is provided about MS in the food sector. A study was conducted in 49 companies in the Caldas Region, Colombia. In CP their relative importance and the dominant management approach were identified (sand cone or trade-off). Regarding SDA, it was analyzed the relationship between their level of development and the company's performance in terms of their CP. The results corroborate diverse theoretical approaches, demonstrating that there is no a single way to address a manufacturing strategy.

Keywords: Manufacturing strategy, operations strategy, competitive priorities, strategic decision areas, sand cone, trade-off.

JEL Classification: D2, M11, L1

\section{Prioridades competitivas e áreas de decisão estratégica na manufatura. Um estudo empírico no setor de alimentos}

\section{ResUMO}

O objetivo deste artigo é contrastar algumas hipóteses relacionadas com as prioridades competitivas ( $\mathrm{PC}$ ) e com as áreas de decisão estratégica $(A D E)$ em estratégia de manufatura (EM). Identificou-se um conjunto ampliado de $\mathrm{PC}$ a respeito dos estudos prévios e contribui-se com evidência empírica no setor de alimentos. Para isso, realizou-se um estudo em 49 empresas no estado de Caldas, Colômbia. Nas PC, foram identificadas sua importância relativa e a abordagem de gestão dominante. Quanto às $A D E$, foi avaliada a relação de seu grau de desenvolvimento com o desempenho das PC. Os resultados corroboram diversos posicionamentos teóricos e demonstram que não existe um único caminho para abordar a estratégia de manufatura.

Palavras-chave: estratégia de manufatura, estratégia de operações, prioridades competitivas, áreas de decisão estratégica, modelos cone de areia e trade-off.

Classificação JEL: D2, M11, L1 


\section{Introducción}

Elárea de manufactura, más allá de cumplir un papel meramente técnico en la fabricación de bienes, hoy en día se entiende como una función gerencial de alto valor estratégico que puede cumplir un papel protagónico en la estrategia global de la compañía (Adamides, 2015; Brown y Blackmon, 2005; Soosay et ál., 2016). Esta perspectiva se ha convertido en un campo de estudio de gran interés académico conocido como estrategia de manufactura o estrategia de operaciones (Boyer et ál., 2005; Miltenburg, 2005; Slack y Lewis, 2011).

La estrategia de manufactura (EM) busca fortalecer las capacidades del sistema de manufactura y apoyar la estrategia organizacional. Los primeros aportes en este campo de estudio se generaron en la Escuela de Negocios de Harvard con los trabajos seminales de Skinner (1969), Hayes y Wheelwright (1984), Swamidass y Newell (1987), entre otros. De dichos trabajos se puede establecer que la EM se refiere a un plan de largo plazo para el sistema de manufactura a partir del cual se definen las acciones que deben ser acometidas para responder a la estrategia de la compañía.

Según Ghazinoory y Khotbesara (2007) existen dos componentes principales que deben ser considerados en la EM: el proceso y el contenido. El proceso se refiere al enfoque metodológico que debe seguirse para formular la estrategia; el contenido aborda el conjunto de decisiones estratégicas para el sistema de manufactura que son necesarias para alcanzar cierto nivel de desempeño (Ibarra y Sarache, 2008). Los aspectos que conforman el contenido constituyen el foco de interés del presente artículo. Desde esta perspectiva, se deben abordar dos tópicos: las prioridades competitivas y las áreas de decisión estratégica (Drohomeretski et ál., 2014).

Las prioridades competitivas se refieren a los objetivos que el sistema de manufactura debe lograr en respuesta a la estrategia global de la organización. El costo, la calidad, las entregas y la flexibilidad constituyen el conjunto de prioridades competitivas de mayor énfasis en la literatura (Kathuria, Porth, Kathuria y Kohli, 2010). No obstante, dadas las exigencias crecientes en los mercados, otras prioridades como la innovación, el servicio y la protección del medio ambiente también deben ganar una posición de relevancia como parte de la EM (Díaz-Garrido, Martín-Peña y Sánchez-López, 2011). En la literatura prevalecen dos enfoques de gestión, con cierta contraposición, para las prioridades competitivas: el modelo secuencial de creación de capacidades o modelo cono de arena (sand cone) y el modelo de intercambio de capacidades o modelo trade-off (Bortolotti et ál., 2015; Dangol et ál., 2015). 
Por su parte, las áreas de decisión estratégica se relacionan con aquellos aspectos estructurales e infraestructurales que deben ser intervenidos para alinear el sistema de manufactura con sus prioridades competitivas. En la literatura, las áreas de decisión típicamente abordadas son: recursos humanos, estructura y controles de la organización, planificación y control de la producción, aprovisionamiento, tecnología de procesos, instalaciones, gestión de la calidad y desarrollo de nuevos productos (da Silveira, 2014; Jia y Bai, 2011; Miltenburg, 2008; Páez y Ovalle, 2013).

Lograr un alto desempeño en el sistema de manufactura implica una articulación adecuada entre las áreas de decisión estratégica y las prioridades competitivas. Sin embargo, de acuerdo con Schroeder y Flynn (2001), la experiencia demuestra que no existe una sola vía para que una empresa alcance una manufactura de alto desempeño; de hecho, existen muchos caminos posibles para orientar la EM (Miltenburg, 2008). En este sentido, la revisión de literatura realizada por Vivares-Vergara et ál. (2015) identificó escasez investigativa y, en particular, la necesidad de mayor evidencia empírica sobre el contenido de la estrategia en sectores particulares de la industria.

Para verificar la existencia de vacíos de conocimiento en estos temas se realizó una revisión de literatura siguiendo los principios propuestos por Bartels (2013) y se utiLizó la metodología Tree of Science para identificar referencias relevantes aplicando la teoría de grafos (Robledo et ál., 2014). Se detectaron 324 referencias y se encontraron varias brechas que motivaron la realización del presente trabajo para aportar evidencias investigativas en torno a las mismas. De un lado, muy pocos estudios han considerado la innovación, el servicio y la protección del medio ambiente como nuevas prioridades competitivas para el sistema de manufactura, lo cual hace que su relación con las áreas de decisión estratégica presente resultados incompletos. De otro lado, los modelos trade-off y cono de arena mantienen un debate en la literatura con evidencias insuficientes para determinar si uno es mejor que el otro. No menos importante resulta mencionar que la mayor parte de las investigaciones se han realizado en países desarrollados y, según Amoako-Gyampah y Acquaah (2008), es relevante hacer investigaciones en países en desarrollo para examinar la robustez de estas teorías.

En atención a lo anterior, el objetivo principal del presente artículo es exponer los hallazgos de un estudio empírico realizado en 49 empresas de alimentos del Departamento de Caldas (Colombia), con el fin de identificar las prioridades competitivas y el grado de desarrollo o fortaleza en las áreas de decisión estratégica. El sector alimentos fue seleccionado por su alto valor estratégico dentro de las apuestas productivas de la 
región cafetera de Colombia, y porque no ha sido objeto de estudio específico en las investigaciones en EM. Dicho sector puede contribuir con la seguridad alimentaria del país (Conpes Social 113, 2007). De hecho, a nivel mundial, se requiere aumentar en $70 \%$ la producción de alimentos hacia el año 2050 (Cardona et ál., 2010) y ello representa oportunidades para el sector. Por otro lado, a pesar de su potencial, el Departamento de Caldas tiene un nivel de desarrollo agroindustrial limitado pues la mayor parte de los productos agrícolas no se transforman sino que se comercializan como materias primas (Cardona y Orrego, 2007).

La investigación se desarrolló mediante encuestas que fueron aplicadas a los jefes de producción de las empresas. Los resultados obtenidos permitieron detectar la existencia de diversas tendencias en la definición de las prioridades competitivas. De hecho, considerando el debate existente en la literatura sobre el cono de arena vs. trade-off no se identificó un modelo dominante para el sector, lo cual es coherente con otros estudios identificados en la revisión de literatura. Con respecto a las áreas de decisión estratégica se identificó un nivel de alineación adecuado con respecto a las prioridades competitivas.

Para su presentación, el presente artículo se ha estructurado de la siguiente manera: en la primera sección se presenta la revisión de la literatura y el planteamiento de las hipótesis de investigación; en la segunda se presenta la metodología utilizada. Los resultados obtenidos son presentados y discutidos en la tercera sección. Finalmente, se establecen las conclusiones generales de la investigación y se plantean futuras líneas de investigación.

\section{Revisión de literatura e hipótesis}

\section{Prioridades competitivas}

Las prioridades competitivas están relacionadas con las preferencias estratégicas a partir de las cuales las organizaciones desean lograr ventaja competitiva (Muzamil y Idris, 2012). Dichas prioridades dependen, fundamentalmente, del sector industrial y del entorno competitivo en el cual la empresa compite (Sarache, Castrillón y Giraldo, 2011). En los últimos años varios autores se han enfocado en el estudio de las prioridades competitivas identificando cuatro clásicas: el costo, la calidad, las entregas y la flexibilidad (Guerrero, 2013; Kathuria et ál., 2010). No obstante, en dependencia del sector y la arena competitiva, la innovación, el servicio y el cuidado del medio ambiente 
también podrían jugar un papel relevante y se han venido posicionando como nuevas prioridades competitivas (Díaz-Garrido et ál., 2011; Miltenburg, 2005).

La tabla 1 corrobora la existencia de ciertas tendencias en las prioridades competitivas de algunos sectores estudiados en contribuciones previas, encontrándose que las más recurrentes son el costo, la calidad, la flexibilidad, las entregas y la innovación; mientras que el servicio y el medio ambiente se han registrado regularmente en los últimos lugares. Por otro lado, se observan algunas diferencias en las prioridades competitivas de acuerdo con el sector, lo cual es el resultado lógico de exigencias que provienen de mercados distintos. Por ejemplo, en el estudio de Prajogo y McDermott (2011), realizado en 190 empresas de servicio, se encontró que las prioridades competitivas más importantes eran la retención de clientes, la entrega, la calidad y la productividad. En contraste, en la investigación de Askar y Mortagy (2007), realizada en 99 compañías egipcias, las prioridades competitivas fueron la calidad, el servicio, las entregas y la innovación.

\section{Tabla 1}

Prioridades competitivas estudiadas en otras investigaciones

\begin{tabular}{|c|c|c|c|c|c|c|c|c|c|}
\hline \multirow[b]{2}{*}{ Sector } & \multirow[b]{2}{*}{ Autores } & \multirow[b]{2}{*}{$\begin{array}{c}\text { País / } \\
\text { Continente }\end{array}$} & \multicolumn{7}{|c|}{ Prioridades competitivas } \\
\hline & & & Costo & Calidad & Flexibilidad & Entregas & Innovación & Servicio & $\begin{array}{c}\text { Medio } \\
\text { ambiente }\end{array}$ \\
\hline $\begin{array}{l}\text { Alimentos } \\
\text { y químico }\end{array}$ & $\begin{array}{l}\text { Shavarini et ál. } \\
(2013)\end{array}$ & Irán & $x$ & $x$ & $x$ & $x$ & $x$ & & \\
\hline \multirow{2}{*}{$\begin{array}{l}\text { Automovi- } \\
\text { lístico }\end{array}$} & $\begin{array}{l}\text { Filho et ál. } \\
\text { (2011) }\end{array}$ & Brasil & $x$ & $x$ & $x$ & $x$ & $x$ & & \\
\hline & $\begin{array}{l}\text { Kim et ál. } \\
(2014)\end{array}$ & Alemania & $x$ & $x$ & $x$ & $x$ & $x$ & & \\
\hline \multirow{2}{*}{ Confección } & $\begin{array}{l}\text { Sarache et ál. } \\
\text { (2011) }\end{array}$ & Colombia & $x$ & $x$ & $x$ & $x$ & & & \\
\hline & $\begin{array}{l}\text { Guerrero } \\
(2013)\end{array}$ & Colombia & $x$ & $x$ & $x$ & $x$ & $x$ & $x$ & \\
\hline \multirow[t]{2}{*}{ Muebles } & $\begin{array}{l}\text { Silva et ál. } \\
(2012)\end{array}$ & Brasil & & & & & & & \\
\hline & $\begin{array}{l}\text { Thürer et ál. } \\
\text { (2013) }\end{array}$ & Brasil & $x$ & $x$ & $x$ & $x$ & $x$ & & \\
\hline \multirow[t]{2}{*}{$\begin{array}{l}\text { Metalme- } \\
\text { cánico }\end{array}$} & $\begin{array}{l}\text { Drohomeretski } \\
\text { et ál. (2014) }\end{array}$ & Brasil & $x$ & $x$ & $x$ & $\mathrm{x}$ & $x$ & & \\
\hline & $\begin{array}{l}\text { Szász y } \\
\text { Demeter (2014) }\end{array}$ & $\begin{array}{l}\text { Europa, } \\
\text { América, Asia }\end{array}$ & $x$ & $x$ & $x$ & $x$ & $x$ & $x$ & $x$ \\
\hline
\end{tabular}

Fuente: elaboración propia. 
Se encontraron pocos estudios que vincularan en la muestra empresas del sector alimentos y, de ellos, ninguno se centró exclusivamente en entender las particularidades de este sector. Así mismo, y a pesar de que la literatura plantea la importancia de identificar las prioridades competitivas para cada contexto regional de competencia, en lo que respecta al territorio colombiano, no se identificaron contribuciones en esta línea de trabajo para el sector. Teniendo en cuenta que no hay una ruta única para que las empresas alcancen la excelencia en manufactura (Schroeder y Flynn, 2001), puede plantearse entonces que, aun dentro de un sector específico, en una región específica, existen empresas con prioridades competitivas diferentes. En atención a lo anterior se planteó la siguiente hipótesis de investigación:

H1a: En la industria de alimentos existen sub-grupos de empresas que se diferencian entre sí desde sus prioridades competitivas.

H1b: En la industria de alimentos, las prioridades competitivas tienden a ser similares por subsectores.

\section{Modelos dominantes en la gestión de las prioridades competitivas}

Dos enfoques se han identificado en la literatura para la gestión y el desarrollo de las prioridades competitivas: el modelo trade-off (ver figura 1a) y el modelo cono de arena (ver figura 1b). En el modelo trade-off se establece que las empresas deben “conceder a una prioridad competitiva un trato preferencial sobre las demás, estableciendo cuales prioridades competitivas deben recibir la mayor inversión en tiempo y recursos" (Boyer y Lewis, 2002, p. 10, traducción propia), en especial porque los sistemas de producción son, con frecuencia, técnicamente restringidos (Da Silveira y Slack, 2001). Los defensores del modelo trade-offargumentan que un sistema de producción con una configuración productiva particular no puede alcanzar un desempeño superior en más de una prioridad competitiva.

En contraposición, como respuesta a las múltiples exigencias que el mercado impone en términos de calidad, costo, flexibilidad y otros objetivos de desempeño, el modelo cono de arena plantea que es posible, a través de proceso de mejora continua secuencial, obtener un desempeño superior y de forma simultánea en más de una prioridad competitiva. Este enfoque, propuesto originalmente por Ferdows y De Meyer (1990), establece una secuencia de mejoramiento de capacidades que parten de la calidad como base para sustentar el fortalecimiento en las demás prioridades competitivas. A partir 
de allí podrían acometerse mejoras de desempeño en las entregas (fiabilidad), la flexibilidad y finalmente en el costo.

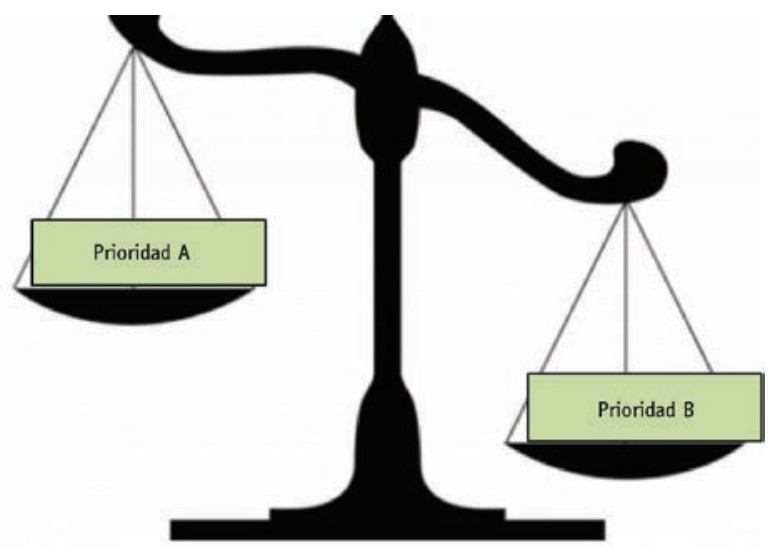

Figura 1a. Trade-off

Fuente: adaptada de Skinner (1974).

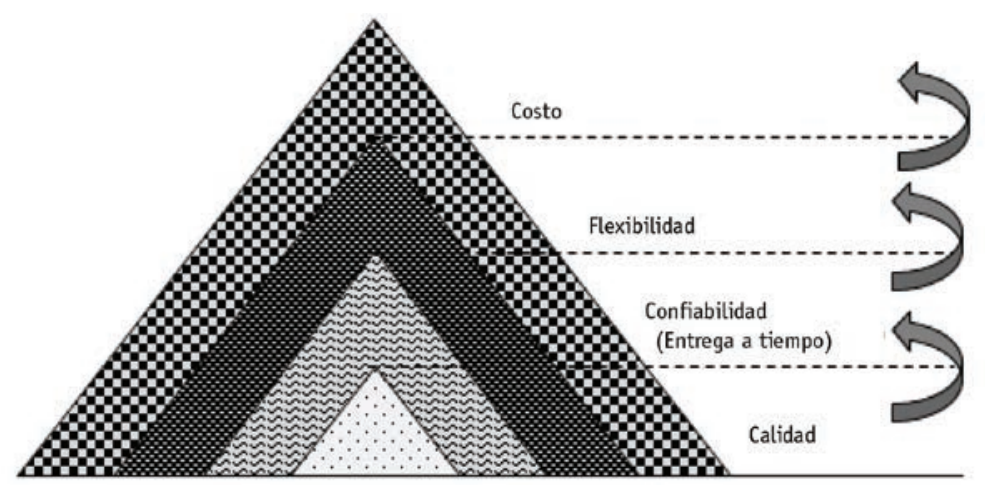

Figura 1b. Cono de arena

Fuente: adaptada de Ferdows y De Meyer (1990).

En general, existen muy pocos estudios que aporten evidencia empírica sobre estos dos enfoques y los resultados obtenidos, en ocasiones contrapuestos, hacen necesaria más investigación en este campo. Desde la perspectiva del cono de arena, el estudio de Schroeder, Shah y Xiaosong Peng (2011), realizado en 189 empresas de manufactura, concluyó que la secuencia de mejora calidad-entregas-flexibilidad-costo no constituyó un patrón constante en todas las empresas analizadas y observaron énfasis en otras 
secuencias. El estudio de Bortolotti, Danese, Flynn y Romano (2015)as described by the sand cone model. Based on the literature, hypotheses relating lean bundles to cumulative performance are proposed. They are tested using a sample of 317 plants in three industries and ten countries, based on structural equation modeling. The results indicate a set of direct and indirect relationships that illustrate: (1, realizado en 317 plantas de varios países, identificó que el modelo cono de arena se ajusta muy bien a enfoques de gestión lean manufacturing, debido a la fortaleza que los procesos de mejora continua ofrecen para la construcción de capacidades en varios objetivos de desempeño. Estos autores evaluaron varias secuencias y encontraron que las empresas que seguían el modelo cono de arena tenían el mejor desempeño.

El estudio de Boyer y Lewis (2002), desarrollado en 110 plantas de Estados Unidos, encontró prevalencia del modelo trade-off sobre el cono de arena. Así mismo, Tawfik Mady (2008), en un estudio en 62 plantas manufactureras en Kuwait, identificó la existencia de trade- off en función del tamaño de la planta. En dicho estudio se encontró que las compañías grandes hacían mayor énfasis en la flexibilidad, mientras que las pequeñas y medianas priorizaban las entregas a tiempo.

Como se evidencia en los trabajos mencionados, en la literatura no existe un consenso en relación con la orientación del sistema de manufactura con respecto a sus prioridades competitivas. Concentrarse en unas pocas de ellas o desarrollar capacidades en todas de forma simultánea, parece ser el resultado de las necesidades y presiones que se ejercen desde cada mercado en particular. Desde esta perspectiva, y para el sector objeto de estudio, se plantean las siguientes hipótesis:

H2a: En la jerarquía dada a las prioridades competitivas predomina un enfoque acumulativo similar al modelo cono de arena.

H2b: En la jerarquía dada a las prioridades competitivas, predomina un enfoque similar al modelo trade-off.

\section{Áreas de decisión estratégica}

Las áreas de decisión estratégica se asimilan a los subsistemas del sistema de manufactura que deben ser intervenidos para mejorar el desempeño en las prioridades competitivas. Éstas se han clasificado en dos grandes categorías: las estructurales y las infraestructurales (Jia y Bai, 2011; Robb y Xie, 2001). Para Martín-Peña y Díaz-Garrido (2011, 
p. 55). Las decisiones de carácter estructural “tienen implicaciones estratégicas, suponen importantes inversiones de capital y afectan a los activos físicos. Su impacto es a largo plazo y son difícilmente reversibles una vez que se han iniciado, debiendo ser supervisadas por la alta dirección". Estas decisiones están relacionadas con la tecnología de procesos, las instalaciones, las fuentes de aprovisionamiento y la capacidad de producción (da Silveira, 2014; Jia y Bai, 2011). Por su parte, “Las decisiones en infraestructura tienen implicaciones operativas que afectan al gasto corriente y que repercuten en el beneficio a corto plazo" (Martín-Peña y Díaz-Garrido, 2011, p. 54). Dentro de esta categoría se encuentran aquellas decisiones relacionadas con el recurso humano, la estructura y controles de la organización, la planificación y el control de la producción, la gestión de la calidad y el desarrollo de nuevos productos (Jia y Bai, 2011; Miltenburg, 2008; Páez y Ovalle, 2013).

Diversas contribuciones sugieren una relación positiva entre las áreas de decisión estratégica y las prioridades competitivas. Slack y Lewis (2002) plantean que el desempeño en las prioridades competitivas depende de la intervención acertada de las áreas de decisión estratégica. Por su parte, Brown, Squire y Blackmon (2007) plantean que en aquellas organizaciones en las que existe un elevado nivel de desempeño, se genera una relación positiva entre las decisiones estructurales de localización, cadena de suministro, tecnología de procesos e instalaciones con varias prioridades competitivas. Así mismo, Swink, Narasimhan y Kim (2005) sugieren que las decisiones de carácter infraestructural y estructural tales como la gestión de proveedores, gestión de la calidad y recursos humanos afectan positivamente el desempeño en algunas prioridades competitivas tales como el costo y la flexibilidad.

En esta misma vía, para Swamidass, Baines y Darlow (2001), el éxito de la estrategia de manufactura está determinado por la manera en que las prioridades competitivas se alinean con las decisiones estratégicas para lograr un alto desempeño en el sistema de manufactura. Ward, Leong y Boyer (1994) agregan que las organizaciones con un alto nivel de desempeño en el mercado crean programas de inversión a largo plazo en las áreas de decisión relacionadas con la tecnología de procesos y el recurso humano, con el fin de lograr capacidades de producción duraderas que garanticen el desarrollo de las prioridades competitivas. La tabla 2 identifica el impacto que desde la intervención en las áreas de decisión estratégica se podrían generar en las prioridades competitivas. 


\section{Tabla 2}

Impacto en las prioridades competitivas

\begin{tabular}{ll}
\hline \multicolumn{1}{c}{ Áreas de decisión } & \multicolumn{1}{c}{ Impacto en las prioridades competitivas } \\
\hline Diseño del producto & Plazo de entrega, calidad y costos \\
Tecnología de procesos & Medio ambiente, flexibilidad \\
Recursos Humanos & Flexibilidad \\
Capacidad & Flexibilidad en productos, costos \\
Instalaciones & Costo, servicio al cliente \\
Integración vertical, aprovisionamiento & Costos, entrega, flexibilidad y servicio \\
Gestión de la calidad & Calidad, costos \\
\hline
\end{tabular}

Fuente: adaptación propia a partir de Ibarra y Sarache (2008).

Para el caso colombiano, Vivares-Vergara et ál. (2014) realizaron un estudio de carácter empírico en empresas manufactureras grandes y medianas, encontrando falencias en las áreas de decisión relacionadas con los procesos de abastecimiento, el layout y la tecnología de procesos. A partir de los resultados obtenidos, se enfatizó en la necesidad de efectuar actividades de mejoramiento en estas áreas, dado el impacto potencial detectado en el costo y las entregas. De acuerdo con la literatura analizada, se espera la existencia de una estrecha relación entre las áreas de decisión y el desempeño en las prioridades competitivas. Por tal motivo se propone la siguiente hipótesis de investigación:

H3: El grado de desarrollo en las áreas de decisión estratégica se relaciona positivamente con el desempeño en las prioridades competitivas de la industria de alimentos.

\section{Metodología}

\section{Población objeto de estudio y muestra}

El estudio se desarrolló en empresas industriales del sector alimentos de todos los tamaños ubicadas en el Departamento de Caldas (Colombia). De acuerdo con los registros oficiales de las cámaras de comercio regionales, se detectaron 65 empresas en este sector ( $57 \%$ micro, $18 \%$ pequeñas, $11 \%$ medianas y $14 \%$ grandes). El trabajo de campo consistió en la aplicación de una encuesta a los jefes de producción de las empresas, para lo cual se intentó un censo en toda la población. Finalmente se recibió información de 49 empresas, lo que equivale a una tasa de respuesta del 75,4\%. La encuesta se 
realizó presencialmente, con diligenciamiento en copia dura (impresa) y con asistencia al encuestado por parte del equipo investigador (dirigida).

\section{Variables utilizadas}

A partir de la revisión de literatura se establecieron las variables que se exponen en la tabla 3. Las prioridades competitivas se midieron de dos formas (importancia y nivel de desempeño). La importancia, a su vez, se dio en dos niveles: importancia dada a cada prioridad competitiva (ordenamiento jerárquico de las variables de primer orden), y calificación en escala Likert de 5 puntos para la importancia de las variables de segundo orden dentro de cada prioridad (1- muy poco importante; 5- muy importante). El desempeño en las prioridades competitivas se midió en escala de 5 puntos (1- muy bajo; 5- muy alto) para cada variable de segundo orden. Por su parte, las áreas de decisión estratégica también se evaluaron en escala Likert de 5 puntos respecto al grado de desarrollo en cada variable (1- muy bajo; 5 - muy alto).

\section{Tabla 3}

Variables de estudio

\begin{tabular}{|c|c|c|}
\hline Temática & $\begin{array}{c}\text { Variables de primer } \\
\text { orden }\end{array}$ & Variables de segundo orden \\
\hline \multirow{7}{*}{$\begin{array}{l}\text { Prioridades } \\
\text { competitivas }\end{array}$} & Costo & $\begin{array}{l}\text { - Capacidad para lograr bajos costos en la producción } \\
\text { - Capacidad de eliminar el desperdicio o despilfarro }\end{array}$ \\
\hline & Calidad & $\begin{array}{l}\text { - Capacidad de entregar productos que cumplan con especificaciones de } \\
\text { clientes } \\
\text { - Capacidad de los procesos para producir productos bajo condiciones } \\
\text { controladas } \\
\text { - Capacidad de producir productos de alto desempeño respecto } \\
\text { competidores }\end{array}$ \\
\hline & Flexibilidad & - Capacidad para fabricar lotes de cualquier tamaño en forma rentable \\
\hline & Innovación & $\begin{array}{l}\text { - Capacidad para fabricar una amplia gama de productos } \\
\text { - Capacidad para hacer cambios en los diseños del producto } \\
\text { - Capacidad para desarrollar e introducir nuevos productos al sistema }\end{array}$ \\
\hline & Entregas & $\begin{array}{l}\text { - Capacidad para proveer tiempos rápidos de entrega } \\
\text { - Capacidad proveer entregas en las fechas y cantidades pactadas }\end{array}$ \\
\hline & Servicio & $\begin{array}{l}\text { - Capacidad de proporcionar servicio al cliente en la preventa } \\
\text { - Capacidad de proporcionar servicio al cliente durante la transacción } \\
\text { - Capacidad de proporcionar servicio al cliente en la postventa }\end{array}$ \\
\hline & Medio ambiente & $\begin{array}{l}\text { - Capacidad del proceso para evitar la contaminación } \\
\text { - Habilidad para diseñar y producir productos amigables con el medio } \\
\text { ambiente }\end{array}$ \\
\hline
\end{tabular}




\begin{tabular}{|c|c|c|}
\hline Temática & $\begin{array}{c}\text { Variables de primer } \\
\text { orden }\end{array}$ & Variables de segundo orden \\
\hline \multirow{7}{*}{$\begin{array}{l}\text { Áreas de } \\
\text { decisión } \\
\text { estratégica }\end{array}$} & Recursos humanos & $\begin{array}{l}\text { - Talento y competencias del personal para el desarrollo de sus } \\
\text { funciones (conocimientos, competencias) } \\
\text { - Satisfacción del personal y clima laboral (sentido de pertenencia, } \\
\text { motivación y clima laboral) } \\
\text { - Políticas y prácticas de gestión humana (sistemas de formación, } \\
\text { selección, compensación, promoción y desarrollo, participación, } \\
\text { fomento del trabajo en equipo, y políticas/programas de salud } \\
\text { ocupacional) }\end{array}$ \\
\hline & $\begin{array}{l}\text { Estructura y } \\
\text { controles }\end{array}$ & $\begin{array}{l}\text { - Estructura organizativa } \\
\text { - Cultura organizacional } \\
\text { - Evaluación del desempeño } \\
\text { - Protección ambiental del proceso y producto }\end{array}$ \\
\hline & $\begin{array}{l}\text { Aprovisionamiento } \\
\text { y distribución }\end{array}$ & $\begin{array}{l}\text { - Gestión de inventarios } \\
\text { - Integración vertical } \\
\text { - Gestión de proveedores (relación y sistema de selección/evaluación) } \\
\text { - Coordinación con la cadena (proveedores, distribuidores, clientes, } \\
\text { otros) }\end{array}$ \\
\hline & $\begin{array}{l}\text { Planeación y } \\
\text { control de la } \\
\text { producción }\end{array}$ & $\begin{array}{l}\text { - Planeación a mediano plazo } \\
\text { - Planeación a corto plazo (programación de producción y personal, } \\
\text { alistamiento de máquinas y flujo de materiales) } \\
\text { - Gestión del mantenimiento (correctivo, preventivo y predictivo) } \\
\text { - Gestión e ingeniería de la calidad }\end{array}$ \\
\hline & $\begin{array}{l}\text { Tecnología de } \\
\text { procesos }\end{array}$ & $\begin{array}{l}\text { - Capacidad de producción } \\
\text { - Know-how del proceso } \\
\text { - Maquinaria y equipo } \\
\text { - Estudio de métodos } \\
\text { - Tecnologías de la información (uso e integración entre los sistemas) }\end{array}$ \\
\hline & Productos & $\begin{array}{l}\text { - Infraestructura tecnológica y de soporte para el diseño de productos } \\
\text { - Evaluación de productos, selección de materiales y elaboración de } \\
\text { fichas técnicas } \\
\text { - El trabajo coordinado entre áreas funcionales para el diseño/ } \\
\text { desarrollo del producto }\end{array}$ \\
\hline & Instalaciones & $\begin{array}{l}\text { - Infraestructura física } \\
\text { - } \quad \text { Calidad de los servicios de apoyo al proceso } \\
\text { - } \quad \text { Tamaño en relación con las perspectivas de crecimiento } \\
\text { - Distribución en planta }\end{array}$ \\
\hline
\end{tabular}

Fuente: elaboración propia.

\section{Pruebas de validez y confiabilidad}

Se realizaron varios análisis para evaluar la validez y confiabilidad de la investigación. En cuanto a la validez de contenido se realizaron tres actividades: 1) revisión de la literatura basada en 124 referencias bibliográficas, de las cuales se identificaron las variables clave a incluir en el estudio; 2 ) evaluación por parte de tres académicos expertos que 
emitieron su concepto en relación con la calidad de la encuesta; 3) prueba piloto con cinco empresas pertenecientes a la población objeto de estudio, con el fin de introducir las mejoras necesarias antes de su aplicación.

La consistencia interna del instrumento de recolección de datos se evaluó mediante coeficientes Alfa de Cronbach para las 7 prioridades y las 7 áreas de decisión, cuyos resultados fueron los vectores $[0,782 ; 0,649 ; 0,528 ; 0,646 ; 0,700 ; 0,570 ; 0,769]$ y $[0,754 ; 0,452 ; 0,523 ; 0,658 ; 0,712 ; 0,832 ; 0,666]$ respectivamente. Nótese que algunos coeficientes son inferiores a 0,6 lo cual resulta normal si se tiene en cuenta que es un nuevo constructo creado para cubrir los vacíos existentes en la literatura, por lo cual es recomendable realizar más refinamientos en investigaciones posteriores. Por esta misma razón, no se utilizaron técnicas estadísticas más avanzadas como análisis de regresión o ecuaciones estructurales.

Finalmente, con el fin de analizar la consistencia de la muestra obtenida y verificar la ausencia de sesgo, se aplicó la prueba no paramétrica U de Mann-Whitney para evaluar si existían diferencias entre las empresas que respondieron la encuesta y las que no lo hicieron, observando el valor de los activos. Con una confiabilidad del 95\% ( $p$-value > $0.05)$, los resultados permiten establecer que no existen diferencias entre estos dos grupos de empresas (ver tabla 4).

\section{Tabla 4}

Prueba de U de Mann-Whitney para la muestra utilizada

\begin{tabular}{lc}
\hline \multicolumn{1}{c}{ Estadístico } & Valor \\
\hline U de Mann-Whitney & 240 \\
W de Wilcoxon & 1566 \\
Z & $-1,867$ \\
P-value & 0,062 \\
\hline
\end{tabular}

Fuente: elaboración propia.

\section{Resultados y discusión}

\section{Prioridades competitivas en la industria de alimentos}

Para contrastar la hipótesis $\mathrm{H} 1 \mathrm{a}$ se realizó un dendograma bajo el método de conglomeración de Ward (ver figura 2). Con el análisis gráfico del dendograma se estableció la posibilidad de clasificar la población en dos clusters. 


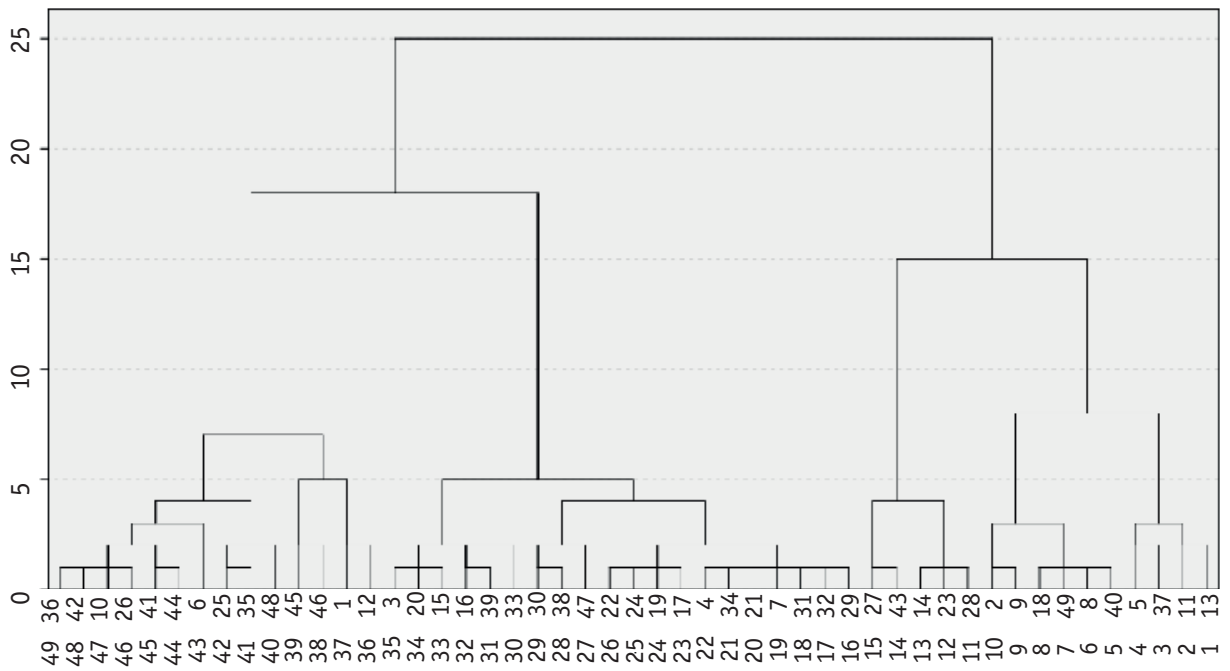

Figura 2. Dendograma

Siguiendo un análisis de cluster de K-medias, se clasificó la población en dos grupos de empresas que presentan diferencias estadísticamente significativas en cuatro de las siete prioridades competitivas analizadas (innovación, entregas, servicio y medio ambiente) (ver tabla 5).

\section{Tabla 5}

ANOVA para la jerarquía dada a las prioridades competitivas en cada cluster

\begin{tabular}{lcccccc}
\hline \multirow{2}{*}{ Variable } & \multicolumn{2}{c}{ Clúster } & \multicolumn{2}{c}{ Error } & \multirow{2}{*}{ F } & Sig. \\
\cline { 2 - 5 } & Media cuadrática & gl & Media cuadrática & gl & & \\
\hline Costo & 3,479 & 1 & 1,998 & 47 & 1,741 & 0,193 \\
Calidad & 1,170 & 1 & 0,935 & 47 & 1,252 & 0,269 \\
Flexibilidad & 1,688 & 1 & 2,857 & 47 & 0,591 & 0,446 \\
Innovación & 20,598 & 1 & 3,400 & 47 & 6,058 & $0,018^{*}$ \\
Entregas & 8,794 & 1 & 2,110 & 47 & 4,168 & $0,047^{*}$ \\
Servicio & 10,776 & 1 & 1,872 & 47 & 5,755 & $0,020^{*}$ \\
Medio ambiente & 146,221 & 1 & 1,733 & 47 & 84,373 & $0,000^{* *}$ \\
\hline
\end{tabular}

* Significativo al 0,05; * Significativo al 0,01.

Fuente: elaboración propia. 
La tabla 6 muestra que la solución obtenida en el análisis de cluster de K-medias logró convergencia en cinco iteraciones. Puede decirse que la estabilidad de dicha solución soporta la confiabilidad de los resultados presentados puesto que en la iteración 5 no se registró ningún cambio en el centro de los clústeres.

\section{Tabla 6}

Historial de iteraciones en el análisis de cluster de K-medias

\begin{tabular}{ccc}
\hline \multirow{2}{*}{ Iteración } & \multicolumn{2}{c}{ Cambiar en centros de clústeres } \\
\cline { 2 - 3 } & 1 & 2 \\
\hline 1 & 4,473 & 4,289 \\
2 & 0,520 & 0,835 \\
3 & 0,463 & 0,839 \\
4 & 0,244 & 0,543 \\
5 & 0,000 & 0,000 \\
\hline
\end{tabular}

Fuente: elaboración propia.

En la tabla 7 se muestra el número de empresas que conforman cada cluster y su composición en relación al tamaño. El cluster uno tiene 33 empresas (en su mayoría micro y pequeñas). El cluster dos tiene 16 empresas (33\% del total).

Tabla 7

Composición de cada cluster

\begin{tabular}{cccccccccccc}
\hline \multirow{2}{*}{ Cluster } & \multirow{2}{*}{$\begin{array}{c}\text { Número de } \\
\text { empresas }\end{array}$} & $\%$ & \multicolumn{8}{c}{ Número de empresas por tamaño } \\
\cline { 4 - 12 } & & Micro & $\%$ & Pequeña & $\%$ & Mediana & $\%$ & Grande & $\%$ \\
\hline 1 & 33 & 67 & 23 & 77 & 7 & 64 & 1 & 25 & 2 & 50 \\
2 & 16 & 33 & 7 & 23 & 4 & 36 & 3 & 75 & 2 & 50 \\
\hline Total & 49 & 100 & 30 & 100 & 11 & 100 & 4 & 100 & 4 & 100 \\
\hline
\end{tabular}

Fuente: elaboración propia.

Ambos clusters difieren en torno a la jerarquía que le otorgan a cada prioridad competitiva (ver tabla 8). A dicha jerarquía se le aplicó un análisis de concordancia mediante el cálculo del coeficiente de concordancia de Kendall (W). Los resultados indican que $W=0,63$ para el clúster 1 y W=0,5 para el clúster 2, lo cual sugiere un nivel de acuerdo aceptable entre las empresas analizadas (Siegel, 1978). Con todo lo anterior, los resultados obtenidos no aportan evidencia suficiente para rechazar la hipótesis H1a. Es decir, en el sector analizado se encontraron al menos dos subgrupos de empresas que se diferencian en la manera como jerarquizan sus prioridades competitivas. 


\section{Tabla 8}

Jerarquía en las prioridades competitivas por cada clúster

\begin{tabular}{clclc}
\hline Orden de importancia & \multicolumn{1}{c}{ Cluster 1 } & Ponderación (\%) & \multicolumn{1}{c}{ Cluster 2 } & Ponderación (\%) \\
\hline $1^{\circ}$ & Calidad & 22,98 & Calidad & 21,0 \\
$2^{\circ}$ & Costo & 21,31 & Costo & 17,4 \\
$3^{\circ}$ & Servicio & 13,62 & Medio ambiente & 17,4 \\
$4^{\circ}$ & Entregas & 13,57 & Servicio & 14,8 \\
$5^{\circ}$ & Innovación & 11,90 & Entregas & 14,6 \\
$6^{\circ}$ & Flexibilidad & 11,55 & Flexibilidad & 8,5 \\
$7^{\circ}$ & Medio Ambiente & 5,07 & Innovación & 6,3 \\
\hline Coeficiente W & & 0,63 & & 0,50 \\
\hline
\end{tabular}

Fuente: elaboración propia.

Para contrastar la hipótesis $\mathrm{H} 1 \mathrm{~b}$ se realizó un análisis de concordancia de Kendall con el fin de verificar si a nivel de sub-sectores (confitería, lácteo, etc.), existía una concordancia aceptable en el ordenamiento o jerarquía dada a las prioridades competitivas. Se seleccionaron aquellos sub-sectores que tuvieran al menos 3 empresas, obteniendo un conjunto final de 8 subsectores con 43 empresas que se sometieron al análisis. Los resultados presentados en la tabla 9 muestran un nivel de concordancia aceptable en 5 de los 8 subsectores analizados $(W \geq 0,5)$, por lo cual no se encontró evidencia suficiente para rechazar la hipótesis $\mathrm{H} 1 \mathrm{~b}$. Este resultado sugiere entonces que las prioridades competitivas tienden a ser similares por subsectores.

Aunque el estudio se llevó a cabo en empresas de un sector específico (alimentos) ubicadas en una región específica (Departamento de Caldas, Colombia), el análisis de la hipótesis 1 permitió identificar diversos subgrupos de empresas con prioridades competitivas diferentes, y sólo se pudieron identificar similitudes en algunos de los subsectores analizados (H1b). Este resultado es consistente con los hallazgos previos de Schroeder y Flynn (2001), quienes basados en estudios adelantados en varios países en el marco del macroproyecto High Performance Manufacturing (HPM), concluyeron que cada fábrica debe establecer su propia estrategia dependiendo del país, el tipo de industria y el contexto competitivo particular. En esta vía, los citados autores afirman que no existe una ruta universalmente buena para todo tipo de empresa.

Desde esta perspectiva, para el sector objeto de estudio se encontró que, aun para una región de análisis muy específica, las empresas tienden a enfatizar en objetivos de manufactura (prioridades competitivas) diferentes. No obstante, debe notarse que, en la 


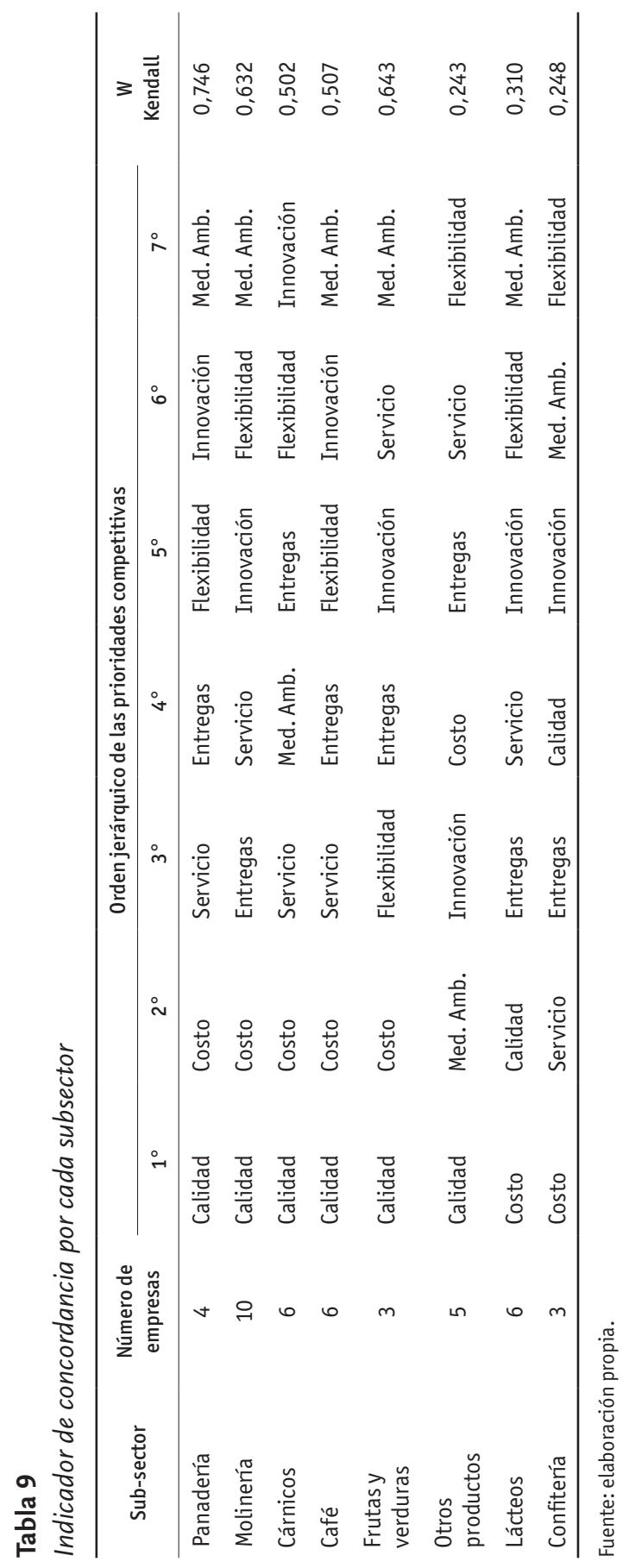


mayor parte de los sub-grupos de empresas analizados, existe una tendencia marcada a ubicar la calidad como principal prioridad competitiva seguida del costo y, por tanto, las diferencias se encuentran en la jerarquía dada a las demás prioridades.

Modelos en la gestión de las prioridades competitivas en la industria de alimentos

Para contrastar la hipótesis $\mathrm{H} 2$ a se realizó un análisis estadístico descriptivo con base en las puntuaciones dadas a cada prioridad competitiva. Los resultados se sintetizan en la tabla 10.

\section{Tabla 10}

Orden de importancia de las prioridades competitivas

\begin{tabular}{clc}
\hline Posición & \multicolumn{1}{c}{ Prioridad } & Ponderación (\%) \\
\hline $1^{\circ}$ & Calidad & 21,9 \\
$2^{\circ}$ & Costo & 19,3 \\
$3^{\circ}$ & Servicio & 14,2 \\
$4^{\circ}$ & Entregas & 14,0 \\
$5^{\circ}$ & Innovación & 10,5 \\
$6^{\circ}$ & Flexibilidad & 10,3 \\
$7^{\circ}$ & Medio Ambiente & 9,9 \\
\hline
\end{tabular}

Fuente: elaboración propia.

Los resultados obtenidos no aportan evidencia para aceptar la hipótesis 2a debido a que el conjunto de empresas no siguen la secuencia de mejoramiento de capacidades propuesta originalmente por Ferdows y De Meyer (1990). En particular, se encontró que sólo el $6 \%$ de las empresas asignan la importancia a sus prioridades de acuerdo con la secuencia propuesta en el modelo cono de arena. De forma complementaria, se evaluó si existía una ventaja de desempeño en las empresas que siguen el cono de arena con respecto a las que no. Con base en la prueba $U$ de Mann-Whitney no se encontraron diferencias significativas entre ambos grupos (ver tabla 11) con una confiabilidad del $95 \%$ ( $p$-value $>0,05)$. 


\section{Tabla 11}

Comparación de medias modelo cono de arena vs. indicador de desempeño

\begin{tabular}{lr}
\hline \multicolumn{1}{c}{ Estadístico } & \multicolumn{1}{c}{ Valor } \\
\hline U de Mann-Whitney & 25,000 \\
W de Wilcoxon & 1106,000 \\
Z & $-1,835$ \\
P-value & 0,066 \\
\hline
\end{tabular}

Fuente: elaboración propia.

Para contrastar la hipótesis $\mathrm{H} 2 \mathrm{~b}$ se tomó como base la importancia dada a las dimensiones de cada prioridad competitiva, calculando así su peso relativo de acuerdo con el puntaje máximo que podría obtener cada una; por ejemplo, existen tres dimensiones en calidad, por lo cual, como máximo, se podrían obtener 15 puntos. De acuerdo con los pesos obtenidos se asignó una variable dummy para clasificar las empresas, así:

1. Existe trade off cuando cada empresa asigna un $90 \%$ o más de los puntos a una o dos de sus prioridades competitivas.

2. No existe trade off cuando la empresa asigna $90 \%$ o más de los puntos a tres 0 más prioridades competitivas simultáneamente.

De esta manera, se obtuvo que un $22 \%$ de las empresas sigue el modelo trade-off, por lo cual no se encontró suficiente evidencia para aceptar la hipótesis H2b. También se evaluó si existía ventaja en el desempeño en las empresas que tienden a seguir el modelo trade-off con respecto a las que no. A través de la prueba U de Mann-Whitney no se encontraron diferencias significativas entre ambos grupos (ver tabla 12).

\section{Tabla 12}

Desempeño entre empresas que aplican el modelo trade-off vs. las que no aplican

\begin{tabular}{lr}
\hline \multicolumn{1}{c}{ Estadístico } & \multicolumn{1}{c}{ Valor } \\
\hline U de Mann-Whitney & 176,500 \\
W de Wilcoxon & 242,500 \\
$Z$ & $-0,779$ \\
P-value & 0,436 \\
\hline
\end{tabular}

Fuente: elaboración propia. 
Adicionalmente, para aquellas empresas que siguen el modelo trade-off no se observó homogeneidad en términos de su enfoque; es decir, de 11 compañías que implementan este modelo en su gestión, el $36 \%$ se enfatizan en la calidad, $36 \%$ en las entregas, $27 \%$ en el servicio y el medio ambiente, $18 \%$ en el costo y ninguna empresa ( $0 \%$ ) en la innovación en productos (ver tabla 13).

\section{Tabla 13}

Prioridades competitivas de mayor énfasis en las empresas que siguen el trade-off

\begin{tabular}{|c|c|}
\hline Prioridad & Porcentaje de empresas (\%) \\
\hline Costo & 18 \\
\hline Calidad & 36 \\
\hline Flexibilidad & 9 \\
\hline Innovación & 0 \\
\hline Entregas & 36 \\
\hline Servicio & 27 \\
\hline Medio Ambiente & 27 \\
\hline
\end{tabular}

Nota: algunas empresas se enfocan en más de una prioridad competitiva por lo cual la suma es superior al 100\%. Fuente: elaboración propia.

Hasta aquí, la investigación exploró hasta dónde las empresas analizadas siguen una gestión de las prioridades competitivas desde la perspectiva del modelo cono de arena o el modelo trade-off. Los hallazgos de la hipótesis 2 indicaron que ninguno de los dos enfoques es prevalente en las empresas analizadas. Además, al comparar las empresas que siguen estos enfoques (independientemente de la cantidad), se encontró que no existían diferencias en el desempeño entre las que se seguían la lógica del cono de arena y las que no, o las que se identificaban con el modelo trade-off y las que no. Así mismo, se observa que el enfoque trade-off es poco prevalente, pues la mayor parte de las empresas (78\%) tiende a privilegiar tres o más prioridades competitivas, seguramente por las exigencias que impone el mercado.

Los resultados presentados deben observarse con cuidado y, en cualquier caso, no restan legitimidad a los modelos expuestos en la literatura, pues ambos se sustentan en antecedentes que aún no llegan a conclusiones unificadas. Por ejemplo, con respecto al modelo cono de arena, Bortolotti et ál. (2015), al evaluar varias secuencias en las prioridades competitivas, concluyeron que este era el que mejor resultados competitivos mostraba en el grupo de empresas analizadas. En contraste, Schroeder, Shah 
y Peng (2011) no encontraron un soporte universal para dicho modelo, mientras que Corbett y Clay y Whybark (2001) encontraron alguna evidencia aunque muy limitada. Así mismo, en el trabajo de Flynn y Flynn (2004) se encontraron algunas diferencias en la secuencia acumulativa en función de cada industria y cada país, por lo cual concluyeron que el desarrollo de capacidades se convierte en una tarea compleja afectada por diversas contingencias interrelacionadas que no deben ser limitadas a una secuencia estandarizada.

Respecto al modelo trade-off, Russell y Millar (2014) realizaron un estudio empírico en el cual encontró falta de evidencia científica para demostrar el predominio del modelo trade-offen la gestión de las prioridades competitivas analizadas. Estos autores señalaron que tales hallazgos son comunes para las empresas de economías menos desarrolladas, ya que las condiciones de competitividad que enfrentan no les permiten enfocarse en una prioridad competitiva en específico. Este hallazgo concuerda con los resultados del presente estudio, aplicado a un grupo de empresas localizadas en un país en vías de desarrollo como Colombia. En la tabla 12 se percibe que, en las empresas que tienden a seguir el enfoque trade-off, existen diversos énfasis entre el conjunto de prioridades competitivas que persiguen. Este hallazgo es consistente con el estudio de da Silveira (2005), quien establece que pueden existir diversos énfasis en una u otra prioridad competitiva de acuerdo con las características de cada compañía y de cada país.

Más allá, teniendo en cuenta este debate, otros autores han promovido una reconciliación considerando que ciertas contingencias estratégicas podrían hacer que un modelo funcione mejor que el otro, por ejemplo, cuando se analizan en función de las fronteras de operación (e.j. Lapréy Scudder, 2004; Liu et ál., 2011; Nand et ál., 2013). Recientemente, Singh et ál. (2015) estudiaron 1438 plantas manufactureras y aportaron evidencias para afirmar que, a los dos enfoques debatidos en la literatura, podrían adicionarse otros cuatro: threshold, average, multiple y uncompetitive. Los hallazgos presentados en esta investigación corroboran que un modelo no es necesariamente mejor que otro. Tales resultados también sugieren la posibilidad de investigar nuevos enfoques para gestionar y desarrollar efectivamente las prioridades competitivas, especialmente en países como Colombia, en el cual las empresas están presionadas para lograr buen desempeño en muchas de ellas, pero quizás no tienen la claridad conceptual suficiente para abordarlo como parte de una estrategia de manufactura bien fundamentada. 


\section{Áreas de decisión estratégica}

Para contrastar la hipótesis H3 se elaboró una matriz de correlaciones con el fin de encontrar las relaciones existentes entre el grado de desarrollo en las áreas de decisión estratégica y el desempeño obtenido en las prioridades competitivas (ver tabla 14).

\section{Tabla 14}

Correlaciones (Spearman) entre las $A D E$ y el desempeño en las PC

\begin{tabular}{lccccccc}
\hline \multicolumn{1}{c}{ Variables de primer nivel } & Costo & Calidad & Flexibilidad & Innovación & Entregas & Servicio & $\begin{array}{c}\text { Med. } \\
\text { Ambiente }\end{array}$ \\
\hline Recursos humanos & $0,394^{* *}$ & $0,348^{*}$ & 0,012 & 0,055 & 0,150 & $0,377^{* *}$ & 0,170 \\
Estructura y controles & $0,379^{* *}$ & $0,340^{*}$ & $-0,094$ & 0,189 & 0,045 & $0,401^{* *}$ & $0,373^{\star *}$ \\
Aprovisionamiento y distribución & 0,180 & $0,321^{*}$ & $-0,159$ & 0,053 & 0,240 & $0,430^{* *}$ & 0,192 \\
Planeación y control de la producción & $0,399^{* *}$ & $0,553^{* *}$ & 0,061 & 0,106 & $0,342^{*}$ & $0,414^{* *}$ & $0,373^{* \star}$ \\
Tecnología de procesos & 0,260 & $0,321^{*}$ & $-0,063$ & 0,239 & 0,086 & 0,206 & 0,101 \\
Productos & $0,356^{*}$ & $-0,071$ & 0,096 & $0,557^{* *}$ & 0,015 & $-0,107$ & $-0,098$ \\
Instalaciones & $0,484^{* *}$ & 0,249 & 0,086 & 0,230 & 0,197 & 0,170 & 0,096 \\
\hline
\end{tabular}

* La correlación es significativa en el nivel 0,05 (1 cola). * * La correlación es significativa en el nivel 0,01 (2 colas).

Fuente: elaboración propia.

Los resultados sugieren que el grado de desarrollo en las áreas de decisión estratégica está relacionado positivamente con el desempeño en las prioridades competitivas, por lo cual se encontró evidencia para soportar la hipótesis H3. Según este estudio, se encontró una correlación significativa para todas las ADE estudiadas con al menos una prioridad competitiva. Sin embargo, debe notarse que la flexibilidad no registró relación significativa con las $A D E$, lo cual sugiere un desajuste de los sistemas de manufactura para contribuir a dicha prioridad competitiva. Estos resultados dan lugar a nuevas hipótesis relacionales que podrían ser trabajadas en investigaciones futuras con métodos más avanzados como el análisis de ecuaciones estructurales.

Este resultado también soporta, refuta y complementa algunas contribuciones de la literatura. Por ejemplo, Ibarra y Sarache (2008) argumentan que las decisiones en desarrollo de productos impactan mayormente en el plazo de entrega, calidad y costo, mientras que la tecnología de procesos impacta en el medio ambiente y la flexibilidad, los recursos humanos en la flexibilidad, las instalaciones en el costo y servicio y el aprovisionamiento en el costo, las entregas, la flexibilidad y el servicio. En el presente estudio, algunas de esas relaciones fueron confirmadas, otras no y, de hecho, se 
identificaron otras no mencionadas por tales autores. Por su parte, Swink, Narasimhan y Kim (2005) encontraron que las decisiones en recursos humanos y aprovisionamiento tienen una relación positiva con el desempeño en la flexibilidad y el costo. Por su parte, el estudio de Vivares-Vergara et ál. (2015) identificó un impacto positivo de las decisiones del desarrollo de nuevos productos, las instalaciones y el aprovisionamiento en el costo y las entregas.

Por consiguiente, es posible afirmar que no existen soluciones universales para afirmar que el énfasis en una determinada área de decisión estratégica impactará en una prioridad competitiva específica, ya que los estudios citados sugieren ciertas contingencias relacionadas con el desempeño en las prioridades competitivas de acuerdo con el área de decisión. Por lo tanto, se puede establecer que la influencia de estas áreas puede estar dependiendo, entre otros factores, del contexto en el cual se encuentre la organización, de la manera en cómo se tomen las decisiones de carácter estratégico y de otras posibles variables que influyan en el desempeño de las mismas, lo cual puede redundar en que se logren relaciones entre áreas y prioridades específicas en algunos casos y en otros no.

\section{Conclusiones}

El presente estudio permitió analizar aspectos relacionados con las prioridades competitivas que persigue la estrategia de manufactura en la industria de alimentos a través de una muestra de empresas colombianas ubicadas en el Departamento de Caldas (Colombia). Se identificaron dos grupos de empresas que se diferencian en función del grado de importancia que conceden al conjunto de prioridades competitivas, lo cual está influido, en gran medida, por el contexto en el que se encuentra cada organización y las características de su mercado objetivo. Por su parte, se evidencia que a nivel de sub-sectores las prioridades competitivas son similares para algunos de ellos, aunque no en todos.

Se puede decir que, aun para un sector particular (alimentos) y una región de análisis muy específica (Caldas), se identifican múltiples sub-grupos de empresas con una jerarquía diferente en las prioridades competitivas que persigue su estrategia de manufactura, aunque puede decirse que la calidad y el costo son las más importantes en la mayoría de dichos sub-grupos. Estos resultados específicos pueden ser utilizados por las entidades que construyen política pública para trazar estrategias de desarrollo en la región. 
Con relación a los modelos de gestión de las prioridades competitivas no se identificó un enfoque dominante entre el trade-off y el cono de arena, y tampoco se encontraron evidencias que sugieran un mayor desempeño competitivo en las empresas que siguen dichos enfoques. Este resultado sugiere la necesidad de seguir avanzando en esta perspectiva de investigación porque en el estado del arte no se identificaron resultados concluyentes. De lo anterior se reafirma que no existe un único camino para gestionar las prioridades competitivas y, por ello, cada empresa debe estructurar su propia ruta con miras a mejorar su desempeño en consonancia con las exigencias del mercado en el cual compite, lo cual implica un reto importante para formular la estrategia de manufactura.

Desde la perspectiva de las áreas de decisión estratégica, se encontraron correlaciones significativas con el desempeño en las prioridades competitivas, en particular, cada área se asoció positivamente con al menos una prioridad competitiva. Es decir, realizar esfuerzos de mejora en las áreas de decisión se relaciona positivamente con el desempeño competitivo en el conjunto de empresas analizadas. Sin embargo, este impacto se genera de manera contingencial en una u otra prioridad, en dependencia de las características de cada organización y otras posibles variables que puedan estar influyendo en el desempeño.

Por tanto, no puede asegurarse que, a partir de esta investigación, las relaciones encontradas puedan extrapolarse a otros sectores u otros países, lo cual sugiere que este asunto debe considerarse como un tema con posibilidades de investigación futura. De hecho, como se demuestra en los resultados y en la revisión de literatura, se concluye que no existe una forma única y universalmente buena para orientar la estrategia de manufactura en cualquier empresa.

\section{Referencias}

Adamides, E. D. (2015). Linking operations strategy to the corporate strategy process: A practice perspective. Business Process Management Journal, 21(2), 267-287. http://doi. org/10.1108/BPMJ-07-2013-0107

Amoako-Gyampah, K. \& Acquaah, M. (2008). Manufacturing strategy, competitive strategy and firm performance: An empirical study in a developing economy environment. International Journal of Production Economics, 111(2), 575-592. http://doi.org/10.1016/j. ijpe.2007.02.030

Askar, M. \& Mortagy, A. K. (2007). Assessing the relative importance of competitive priorities in Egyptian Companies. SAM Advanced Management Journal, 72(3), 35-46. 
Bartels, E. M. (2013). How to perform a systematic search. Best Practice y Research Clinical Rheumatology, 27, 295-306. http://doi.org/10.1016/j.berh.2013.02.001

Bortolotti, T., Danese, P., Flynn, B. \& Romano, P. (2015). Leveraging fitness and lean bundles to build the cumulative performance sand cone model. International Journal of Production Economics, 162, 227-241. http://doi.org/10.1016/j.ijpe.2014.09.014

Boyer, K. K. \& Lewis, M. W. (2002). Competitive priorities: Investigating the need for trade-offs in operations strategy. Production and Operations Management, 11(1), 9-20. https:// doi.org/10.1111/j.1937-5956.2002.tb00181.x

Boyer, K. K., Swink, M. \& Rosenzweig, E. D. (2005). Operations strategy research in the POMS journal. Production and Operations Management, 14(4), 442-449. https://doi.org/10.1111/j.1540-5915.1994.tb00808.x

Brown, S. \& Blackmon, K. (2005). Aligning manufacturing strategy and business-level competitive strategy in new competitive environments: The case for strategic resonance. Journal of Management Studies, 42(4), 793-815. http://doi.org/10.1111/j.1467-6486.2005.00519.x

Brown, S., Squire, B. \& Blackmon, K. (2007). The contribution of manufacturing strategy involvement and alignment to world-class manufacturing performance. International Journal of Operations y Production Management, 27(3), 282-302. http://doi. org/10.1108/01443570710725554

Cardona, C. A. y Orrego, C. E. (2007). La agroindustria y su potencial en Caldas. En La logística del transporte. Un elemento estratégico en el desarrollo agroindustrial (pp. 143-174). Manizales: Gobernación de Caldas.

Cardona, C. A., Orrego, C. E. y Tamayo, J. A. (2010). Análisis de la agroindustria caldense y sus perspectivas de desarrollo. Manizales: Universidad Nacional de Colombia, Sede Manizales.

Conpes Social 113. (2007). Política nacional de seguridad alimentaria y nutricional, PSAN. Bogotá: Departamento Nacional de Planeación.

Corbett, L. M. \& Clay Whybark, D. (2001). Searching for the sandcone in the GMRG data. International Journal of Operations y Production Management, 21(7), 965-980. https://doi. org/10.1108/01443570110393441

Da Silveira, G. J. C. (2005). Improving trade-offs in manufacturing: Method and illustration. International Journal of Production Economics, 95(1), 27-38. http://doi.org/http:// dx.doi.org/10.1016/j.ijpe.2003.10.023

Da Silveira, G. J. C. (2014). An empirical analysis of manufacturing competitive factors and offshoring. International Journal of Production Economics, 150, 163-173. http://doi. org/http://dx.doi.org/10.1016/j.ijpe.2013.12.031

Da Silveira, G. \& Slack, N. (2001). Exploring the trade-off concept. International Journal of Operations and Production Management, 21(7), 949-964. https://doi. org/10.1108/01443570110393432 
Dangol, R., Bahl, M. \& Karpak, B. (2015). Timing cooperative relationships with sequential capability development process to reduce capability development trade-offs. International Journal of Production Economics, 169, 179-189. http://doi.org/10.1016/j.ijpe.2015.07.014

Díaz-Garrido, E., Martín-Peña, M. L. \& Sánchez-López, J. (2011). Competitive priorities in operations: Development of an indicator of strategic position. CIRP Journal of Manufacturing Science and Technology, 4(1), 118-125. http://doi.org/10.1016/j.cirpj.2011.02.004

Drohomeretski, E., Gouvea da Costa, S. E., Pinheiro de Lima, E. \& Garbuio, P. (2014). Lean, six sigma and lean six sigma: An analysis based on operations strategy. International Journal of Production Research, 52(3), 804-824. https://doi.org/10.1080/00207543.2013.842015

Ferdows, K. \& De Meyer, A. (1990). Lasting improvements in manufacturing performance: In serach of a new theory. Journal of Operations Managment, 9(2), 168-184. https://doi. org/10.1016/0272-6963(90)90094-T

Filho, A., Nogueira, E. \& Bento, P. (2011). Análise das estratégias de produção de seis montadoras de motores para automóveis. Gestão e Produção, 18(3), 603-618. https://doi. org/10.1590/S0104-530X2011000300012

Flynn, B. B. \& Flynn, E. J. (2004). An exploratory study of the nature of cumulative capabilities. Journal of Operations Management, 22(5), 439-457. https://doi.org/10.1016/j. jom.2004.03.002

Ghazinoory, S. \& Khotbesara, R. M. (2007). The manufacturing strategy formation process case study of six Iranian manufacturing companies. In IEEM 2007: International Conference on Industrial Engineering and Engineering Management (pp. 733-737). http://doi. org/10.1109/IEEM.2007.4419287

Guerrero, G. (2013). Las prioridades competitivas de manufactura y su papel en la competitividad local: proyecciones del sector industrial de Ibagué a través del análisis multivariante. Revista $E A N, 75,26-37$.

Hayes, R. H. \& Wheelwright, S. C. (1984). Restoring our competitive edge: competing through manufacturing. New York: Wiley.

Ibarra, S. y Sarache, W. (2008). Dirección de la producción: su papel estratégico en la competitividad empresarial. En F. Becerra (ed.), Gestión de la producción : una aproximación conceptual (1a ed, p. 15). Manizales: Universidad Nacional de Colombia.

Jia, G. Z. \& Bai, M. (2011). An approach for manufacturing strategy development based on fuzzyQFD. Computers \& Industrial Engineering, 60(3), 445-454. https://doi.org/10.1016/j. cie.2010.07.003

Kathuria, R., Porth, S. J., Kathuria, N. N. \& Kohli, T. K. (2010). Competitive priorities and strategic consensus in emerging economies: Evidence from India. International Journal of Operations \& Production Management, 30(8), 879-896. https://doi.org/10.1108/01443571011068207 
Kim, Y. H., Sting, F. J. \& Loch, C. H. (2014). Top-down, bottom-up, or both? Toward an integrative perspective on operations strategy formation. Journal of Operations Management, 32(7-8), 462-474. https://doi.org/10.1016/j.jom.2014.09.005

Lapré, M. A. \& Scudder, G. D. (2004). Performance improvement paths in the U.S. airline industry: Linking trade-offs to asset frontiers. Production and Operations Management, 13(2), 123-134. https://doi.org/10.1111/j.1937-5956.2004.tb00149.x

Liu, N., Roth, A. V. \& Rabinovich, E. (2011). Antecedents and consequences of combinative competitive capabilities in manufacturing. International Journal of Operations \& Production Management, 31(12), 1250-1286. https://doi.org/10.1108/01443571111187448

Martín-Peña, M. y Díaz-Garrido, E. (2011). Un análisis del impacto de la estrategia de producción en los resultados empresariales. Revista Europea de Dirección y Economía de la Empresa, 20(2), 53-72.

Miltenburg, J. (2005). Manufacturing Strategy: How to formulate and implement a winning plan. New York: Productivity Press.

Miltenburg, J. (2008). Setting manufacturing strategy for a factory-within-a-factory. International Journal of Production Economics, 113(1), 307-323. https://doi.org/10.1016/j. ijpe.2007.09.001

Muzamil, M. \& Idris, F. (2012). Competitive priorities in Malaysian service industry. Business Strategy Series, 13(6), 263-273. https://doi.org/10.1108/17515631211286100

Nand, A. A., Singh, P. \& Power, D. (2013). Testing an integrated model of operations capabilities: An empirical study of Australian airlines. International Journal of Operations \& Production Management, 33(7), 887-911. https://doi.org/10.1108/IJ0PM-12-2011-0484

Páez, Y. y Ovalle, A. M. (2013). Análisis de los sistemas de programación de la producción en la gran empresa de la región centro sur de Caldas, Colombia. Ingenieria industrial, actualidad y nuevas tendencias, 3(10), 91-98.

Prajogo, D. I. \& McDermott, P. (2011). Examining competitive priorities and competitive advantage in service organisations using importance-performance analysis matrix. Managing Service Quality: An International Journal, 21(5), 465-483. https://doi. org/10.1108/09604521111159780

Robb, D. J. \& Xie, B. (2001). A survey of manufacturing strategies in China-based enterprises. International Journal of Production Economics, 72(2), 181-199. https://doi.org/10.1016/ S0925-5273(00)00147-X

Robledo, S., Osorio, G. A., y López, C. (2014). Networking en pequeña empresa: una revisión bibliográfica utilizando la teoria de grafos. Revista Vínculos, 11(2), 6-16. https://doi. org/10.14483/issn.2322-939X

Russell, S. N. \& Millar, H. H. (2014). Competitive priorities of manufacturing firms in the Caribbean. IOSR Journal of Business and Management, 16(10), 72-82. 
Sarache, W., Castrillón, 0. y Giraldo, J. (2011). Prioridades competitivas para la industria de la confección. Estudio de caso. Cuadernos de Administración, 24(43), 89-110.

Schroeder, R. G. \& Flynn, B. B. (2001). High performance manufacturing. Global perspectives. New York: John Wiley \& Sons.

Schroeder, R. G., Shah, R. \& Xiaosong Peng, D. (2011). The cumulative capability "sand cone" model revisited: A new perspective for manufacturing strategy. International Journal of Production Research, 49(16), 4879-4901. https://doi.org/10.1080/00207543.2010.509116

Shavarini, S.K., Salimian, H., Nazemi, J. \& Alborzi, M. (2013). Operations strategy and business strategy alignment model (case of Iranian industries). International Journal of Operations \& Production Management, 33(9), 1108-1130. https://doi.org/10.1108/ IJOPM-12-2011-0467

Siegel, S. (1978). Estadística no paramétrica aplicada a las ciencias de la conducta. México D.F.: Editorial Trillas.

Silva, E. M., Santos, F. C. \& Castro, M. (2012). Análise das relações entre estratégia de produção, práticas e desempenho operacional. Produção, 22(3), 502-516. http://doi.org/10.1590/ S0103-65132012005000009

Singh, P., Wiengarten, F., Nand, A. \& Betts, T. (2015). Beyond the trade-off and cumulative capabilities models: Alternative models of operations strategy. International Journal of Production Research, 53(13), 4001-4020. http://doi.org/10.1080/00207543.2014.983277

Skinner, W. (1974). The Focused Factory. New approach to managing manufacturing sees our productivity crisis as the problem of how to compete. Harvard Business Review, 3(52), 113-121.

Skinner, W. (1969). Manufacturing - missing link in corporate strategy. Harvard Business Review, 47(3), 136-145.

Slack, N. \& Lewis, M. (2011). Operations Strategy (3rd ed). Edinburgh: Pearson Prentice Hall.

Slack, N. \& Lewis, M. (2002). Operations strategy (1st ed). New Jersey: Prentice Hall.

Soosay, C., Nunes, B., Bennett, D., Sohal, A., Jabar, J. \& Winroth, M. (2016). Strategies for sustaining manufacturing competitiveness. Journal of Manufacturing Technology Management, 27(1), 6-37. http://doi.org/10.1108/JMTM-04-2014-0043

Swamidass, P. M., Baines, T. \& Darlow, N. (2001). The role of manufacturing and marketing managers in strategy development: Lessons from three companies. International Journal of Operations \& Production Management, 21(7), 933-948. http://doi.org/https://doi. org/10.1108/01443570110393423

Swamidass, P. M. \& Newell, W. T. (1987). Manufacturing strategy, environmental uncertainty and performance: A path analytic model. Management Science, 33(4), 509-524. http:// doi.org/10.1287/mnsc.33.4.509 
Swink, M., Narasimhan, R. \& Kim., S. W. (2005). Manufacturing practices and strategy integration: Effects on cost efficiency, flexibility, and market-based performance. Decision Sciences, 36(3), 427-457. https://doi.org/10.1111/j.1540-5414.2005.00079.x

Szász, L. \& Demeter, K. (2014). How do companies lose orders? A multi-country study of internal inconsistency in operations strategies. Operations Management Research, 7(3-4), 99-116. http://doi.org/10.1007/s12063-014-0091-z

Tawfik M., M. (2008). The impact of plant size and type of industry on manufacturing competitive priorities: An empirical investigation. Competitiveness Review: An International Business Journal, 18(4), 351-366. http://doi.org/10.1108/10595420810920824

Thürer, M., Godinho F., M., Stevenson, M. \& Fredendall, L. D. (2013). Competitive priorities of small manufacturers in Brazil. Industrial Management \& Data Systems, 113(6), 856-874. http://doi.org/10.1108/IMDS-01-2013-0049

Vivares-Vergara, J. A., Castaño-González, L. E. y Sarache, W. (2015). Estrategia de operaciones: una revisión sistemática de literatura. En II Congreso Internacional Industria y Organizaciones - Logística, Innovación y Desarrollo Tecnológico (pp. 1-15). Bogotá.

Vivares-Vergara, J. A., Sarache-Castro, W. A. \& Naranjo-Valencia, J. C. (2014). The content of manufacturing strategy: A case study in Colombian industries. Dyna, 81(183), 140-147. http://doi.org/http://dx.doi.org/10.15446/dyna.v81n183.37672

Vivares-Vergara, J. A., Sarache, W. A. y Naranjo-Valencia, J. C. (2015). Estrategia de manufactura: explorando el contenido y el proceso. Información Tecnológica, 26(3), 87-98. http:// doi.org/http://dx.doi.org/10.4067/S0718-07642015000300013

Ward, P., Leong, G. K. \& Boyer, K. K. (1994). Manufacturing proactiveness and performance. Decision Sciences, 25(3), 337-358. https://doi.org/10.1111/j.1540-5915.1994.tb00808.x 